\title{
Saúde autopercebida e qualidade de vida de homens participantes de intervenção psicoeducativa para idosos
}

\author{
Lilian Maria Borges - Universidade Federal Rural do Rio de Janeiro, Rio de Janeiro, Brasil \\ Eliane Maria Fleury Seidl - Universidade de Brasilia, Brasilia, Brasil
}

\begin{abstract}
Resumo
Este trabalho investigou percepções de saúde e de envelhecimento ativo entre homens idosos, assim como seus níveis de qualidade de vida antes e após intervenção psicoeducativa em grupo destinada à promoção de autocuidado. Treze homens de 62 a 78 anos, casados e aposentados, participaram de nove encontros temáticos. Os dados foram obtidos mediante entrevistas individuais semiestruturadas e aplicação dos questionários para aferir qualidade de vida WHOQOL-OLD e WHOQOL-BREF. Os resultados evidenciaram que os idosos, em sua maioria, mantinham estilos de vida ativos, avaliavam positivamente o estado pessoal de saúde e concebiam saúde em uma perspectiva biopsicossocial, aspectos que se mostraram fortalecidos após a intervenção. Houve estabilidade nos escores de qualidade de vida, com diferença significativa $(\mathrm{p}<0,05)$ para o domínio participação social do WHOQOL-OLD. O estudo forneceu um modelo de intervenção para fortalecer o envelhecimento bem-sucedido entre homens idosos.
\end{abstract}

Palavras-chave: Idosos; Homens; Qualidade de vida; Saúde.

\section{Self-perceived health and quality of life of men participating in psychoeducational intervention for elderly}

\begin{abstract}
This study investigated perceptions of health and active aging among elderly men, as well as their levels of quality of life before and after group psychoeducational intervention designed to promote self-care. Thirteen men (from 62 to 78 years old, married and retired) participated in nine thematic meetings. Data were collected through semi-structured interviews and application of the questionnaires WHOQOL-OLD and WHOQOL-BREF to assess quality of life. Results showed that the elderly mostly kept active lifestyles, self-evaluations had been positive for personal health and health was conceived in a biopsychosocial perspective, aspects that were strengthened after the intervention. There was stability in scores for quality of life, with a significant difference $(p<0.05)$ for the social participation domain of the WHOQOL-OLD. The study provided a model of intervention to strengthen successful aging among elderly men.
\end{abstract}

Keywords: Aged; Men; Quality of life; Health.

Salud autopercibida y calidad de vida de hombres participantes de intervención psicoeducativa para ancianos

\begin{abstract}
Resumen
Este trabajo investigó percepciones de la salud y envejecimiento activo entre hombres ancianos, así como sus niveles de calidad de vida antes y después de intervención psicoeducativa en grupo destinado a la promoción de autocuidado. Trece hombres entre 62 y 78 años, casados y jubilados, participaron de nueve encuentros temáticos. Los datos fueron obtenidos mediante entrevistas individuales semiestructuradas y aplicación de cuestionarios para medir cualidad de vida WHOQOL-OLD e WHOQOL-BREF. Los resultados evidenciaron que los ancianos en su mayoría mantenían estilos de vida activa, evaluaban positivamente su condición personal y concebían su salud en una perspectiva biopsicosocial, aspectos que se mostraron fortalecidos después de la intervención. Hubo estabilidad en la puntuación de calidad de vida con diferencia significativa $(\mathrm{p}<0,05)$ para el dominio participación social del WHOQOL-OLD. El estudio proporcionó un modelo de intervención para fortalecer el envejecimiento bien sucedido entre hombres ancianos.

Palabras-clave: Adultos mayores; Hombres; Calidad de vida; Salud.
\end{abstract}

O envelhecimento populacional constitui um dos principais desafios da atualidade. Os progressos tecnológicos e as novas políticas públicas acarretaram o declínio das taxas de mortalidade infantil, a diminuição dos níveis de fecundidade e o aumento da longevidade, o que resultou, em escala mundial, no aumento progressivo e acentuado da parcela da população com idade superior a 60 anos (Camarano, 2002; Netto, 2002; WHO, 2005). Nesse cenário, respaldados na visão da velhice como uma fase do ciclo de vida que pode ser acrescida de bem-estar e qualidade, programas e serviços têm

Disponivel em wnw.scielo.br sido dirigidos aos idosos com vistas a prevenir doenças e incapacidades e a oferecer-lhes atividades orientadas para a promoção da saúde (Assis, Hartz, \& Valla, 2004). A atenção recai, sobretudo, sobre aqueles que vivenciam o processo de envelhecimento em contextos de maior vulnerabilidade, incluindo o acometimento por doenças e a carência de recursos sociais e financeiros (Silva, Lima, \& Galhardoni, 2010).

Os programas (psico)educativos, mediante o emprego de variadas estratégias metodológicas, buscam promover comportamentos de autocuidado e 
maior autonomia entre os idosos participantes, além de aumentar seus conhecimentos acerca de mudanças típicas do envelhecimento e de formas de prevenção e controle de patologias prevalentes na velhice (Assis, Hartz, Pacheco, \& Valla, 2009; Carvalho, Fonseca, \& Pedrosa, 2004; Diogo, Ceolim, \& Cintra, 2000; Gonçalves \& Schier, 2005; Lahm-Vieira \& Boeckel, 2012; Morais, 2009). Em encontros periódicos para a discussão e a reflexão de diferentes temas, a fala dos participantes é valorizada e eles são estimulados a tomar decisões e a realizar mudanças em seus cotidianos. Complementarmente a participação nos pequenos grupos, os idosos, em alguns trabalhos, recebem ainda orientações e recomendações individuais, por meio de contato pessoal e/ ou de material gráfico (Bode \& Ridder, 2007; Cervato, Derntl, Latorre, \& Marucci, 2005; Dapp, Anders, von Renteln-Kruse, \& Meier-Baumgartner, 2005; Domínguez \& Juste, 2003).

A elucidação dos fatores motivacionais que favorecem a participação dos idosos em programas de educação em saúde requer a exploração de significados e valores na perspectiva deles próprios. Miller e Iris (2002) investigaram atitudes e crenças de 45 idosos, distribuídos em seis grupos focais, em relação a bem-estar, autocuidado e benefícios de integrar atividades de promoção da saúde. Os participantes descreveram saúde como um conceito multidimensional em que bem-estar físico e bem-estar psicológico foram vistos como interdependentes. Os componentes do "ser saudável" incluíram independência funcional, autocuidado, visão positiva, crescimento pessoal e contribuição social. As estratégias mencionadas para promover estilos de vida saudáveis foram categorizadas em comportamentos de saúde, nas dimensões física (p. ex. praticar exercícios físicos) e psicossocial (p. ex., estabelecer prioridades, expressar sentimentos), bem como em engajamento, nas dimensões cognitiva (p. ex., ter aulas) e interpessoal (p. ex., atuar como voluntário).

$\mathrm{Na}$ velhice, a promoção da saúde e a melhoria da qualidade de vida têm estreita relação com o envelhecimento bem-sucedido. O termo envelhecimento ativo foi adotado pela Organização Mundial de Saúde-OMS, no final da década de 1990, para designar "o processo de otimização das oportunidades de saúde, participação e segurança, com o objetivo de melhorar a qualidade de vida à medida que as pessoas ficam mais velhas" (WHO, 2005 , p. 13). Por conseguinte, é esperado que os idosos não somente alcancem uma vida longa, mas que possam estar fisicamente ativos e tenham asseguradas suas necessidades de participação social, proteção, segurança e cuidados.

Além da manutenção de um bom estado de saúde física e do baixo risco de doenças, envelhecer bem está associado à participação na comunidade, envolvimento ativo com a vida, independência e manutenção da autonomia (Netto, 2002). Uma velhice bem-sucedida, em linhas gerais, resulta do equilíbrio entre as limitações e as potencialidades do indivíduo em seu processo de envelhecimento (Neri, Yassuda, \& Cachioni, 2004).

De acordo com a OMS, vários são os fatores determinantes da saúde que, em interação, contribuem para o envelhecimento ativo. Esses fatores podem ser organizados em cultura e gênero; fatores relacionados aos sistemas de saúde e serviço social; fatores comportamentais, como ausência de tabagismo, atividade física e alimentação saudável; aspectos pessoais, como genética e capacidade cognitiva; ambiente físico, como moradia segura, água potável, ar puro e alimentos seguros; ambiente social, como apoio social e educação e fatores econômicos, como renda e trabalho (WHO, 2005). Pode-se concluir que o tipo de velhice a ser vivenciado dependerá de fatores genético-biológicos, da história individual, bem como do contexto social e histórico-cultural.

Um estudo conduzido por Dalla Vecchia, Ruiz, Bocchi e Corrente (2005) revelou que, para os idosos entrevistados, a percepção de qualidade de vida na velhice pode ser organizada nas seguintes categorias: preservação dos relacionamentos interpessoais, manutenção de uma boa saúde, manutenção do equilíbrio emocional, lazer, trabalho com prazer, vivência da espiritualidade, prática da retidão e da caridade, acesso ao conhecimento e moradia em locais favoráveis.

$\mathrm{Na}$ conceituação de qualidade de vida, a subjetividade e a abordagem multidimensional têm relevância amplamente reconhecida. Em estudo de revisão deste construto, Seidl e Zannon (2004) destacam a percepção da pessoa como fundamental na apreciação de seu estado de saúde e do seu contexto de vida, bem como ressaltam que o indivíduo deve ser requerido a avaliar a sua situação pessoal em várias dimensões.

Medidas de qualidade de vida têm sido utilizadas no estudo do envelhecimento bem-sucedido e na avaliação da eficácia, eficiência e impacto de intervenções e serviços que objetivam ajudar os idosos a permanecerem independentes e autônomos. As intervenções avaliadas têm por finalidade melhorar o bem-estar e elevar os níveis de qualidade de vida dos participantes, mediante o favorecimento, nessa fase da vida, de 
independência, participação social, preservação de capacidades cognitivas, hábitos saudáveis e/ou redução de ansiedade e depressão (Ascensão, 2011; Castro, Tahara, Rebelatto, Driusso, Aveiro, \& Oishi, 2007; Vitorino, 2012).

Os grupos para promoção de saúde e qualidade de vida na velhice destacam-se pelo predomínio da participação feminina, que, em geral, chega à quase totalidade. Por conseguinte, observa-se a necessidade de estratégias motivadoras para a maior representação masculina neste tipo de intervenção. $\mathrm{Na}$ experiência com grupos de apoio formados com homens portadores ou sobreviventes do câncer de próstata, Oliffe, Gerbrandt, Bottorff e Hislop (2010) confirmaram a viabilidade de espaços em que os homens possam discutir suas experiências particulares de adoecimento, obter orientações e refletir sobre os benefícios do autocuidado.

Considerando o panorama apresentado, o objetivo do presente estudo consistiu em identificar a saúde autopercebida e a concepção de envelhecimento saudável em homens idosos, bem como em avaliar suas percepções da própria qualidade de vida, em níveis globais e em domínios específicos, antes e após a participação em uma intervenção psicoeducativa em grupo.

\section{Método}

\section{Participantes}

A pesquisa teve a participação de treze homens, com idades entre 62 e 78 anos $(M=69,5)$. Como características comuns, esses idosos estavam aposentados, eram alfabetizados, mantinham relações conjugais estáveis há, no mínimo, quinze anos e apresentavam pelo menos uma doença crônica, sendo as mais frequentes doenças cardiovasculares e diabetes mellitus. Por outro lado, possuíam distintos níveis de escolaridade, com predomínio do nível médio (53,8\%). Majoritariamente, declararam-se como católicos (53,8\%), mas havia também praticantes de religiões evangélicas e adeptos do espiritismo. Em relação à renda familiar, verificou-se um predomínio de rendimento mensal equivalente ou superior a quatro salários mínimos (53,8\%).

O recrutamento dos participantes ocorreu em um Centro de Convivência para Idosos (CCI) vigente em uma instituição de ensino superior privada do Distrito Federal. Como parte das atividades regulares do CCI, os idosos tinham acesso a atividades físicas, artístico-culturais e socioeducativas, as quais eram realizadas durante a semana em horários diversos e de forma gratuita.

\section{Instrumentos}

Questionário de Avaliação da Saúde do Homem Idoso - QUASHI, construído para os fins da pesquisa, está estruturado em cinco partes, com 32 questões abertas e fechadas destinadas à caracterização sociodemográfica e à obtenção de medidas de autorrelato sobre avaliação da saúde e doenças autorreferidas, busca e utilização de serviços de saúde, comportamentos preventivos e definição de metas de saúde. O QUASHI foi elaborado devido ao contexto brasileiro ser ainda carente de instrumentos validados para mensuração de comportamentos de autocuidado na população idosa. As respostas obtidas mediante o uso de escalas likert não possibilitaram o alcance de um escore global e, por conseguinte, as propriedades psicométricas do questionário ainda não foram testadas, embora este tenha sido alvo de um estudo piloto que avaliou sua clareza e objetividade. Para as respostas às questões abertas do instrumento, deve ser efetuada análise de conteúdo.

Os dados do QUASHI utilizados no presente trabalho correspondem a questões da segunda parte do instrumento que visam investigar a concepção de saúde do participante, avaliar seu estado de saúde atual em escala likert de cinco pontos e comparar a sua saúde com a de outros idosos e com a própria saúde em fases anteriores do ciclo de vida.

World Health Organization Quality of Life -WHOQOL, instrumento elaborado pela OMS para aferir medidas genéricas de qualidade de vida, que, em sua versão completa, abarca 100 itens (WHOQOL-100). O WHOQOL-BREF consiste na versão abreviada do questionário e compreende 26 questões, sendo 24 delas representativas de quatro domínios: físico, psicológico, relações sociais e meio ambiente. As respostas às questões de cada domínio podem variar de um a cinco. Três itens foram formulados de forma negativa e devem ser invertidos, já que resultados mais elevados caracterizam uma melhor qualidade de vida. Uma versão em português do instrumento foi adaptada e validada por Fleck et al. (2000). O questionário apresenta características satisfatórias de consistência interna, validade e confiabilidade.

WHOQOL-OLD, instrumento para avaliação da qualidade de vida especificamente de adultos idosos. Seus 24 itens são respondidos em escala likert de cinco pontos, distribuídos em seis facetas: funcionamento do sensório; autonomia; atividades passadas, presentes e futuras; participação social; morte e morrer; e intimidade. Cada uma dessas facetas possui quatro itens e, para todas elas, o escore dos valores possíveis oscila 
entre quatro a vinte. Os escores das seis facetas ou os valores dos 24 itens combinados produzem o escore global. A versão brasileira deste módulo do WHOQOL foi validada por Fleck, Chachamovich e Trentini (2006), tendo evidenciado bom desempenho psicométrico na investigação da qualidade de vida em idosos.

\section{Procedimentos de coleta de dados}

De acordo com a Resolução 466/2012 do Conselho Nacional de Saúde, o projeto de pesquisa foi primeiramente apresentado a um Comitê de Ética em Pesquisa. Após sua aprovação, os objetivos e os procedimentos gerais do trabalho planificado foram apresentados, em grupo, a homens idosos que estavam vinculados a um centro de convivência de uma universidade privada do DF. Ligações telefônicas foram efetuadas para aqueles que consentiram em integrar a pesquisa, visando ao agendamento de horários para os encontros individuais de aplicação dos instrumentos.

As entrevistas foram realizadas em salas de atendimento de uma clínica-escola de psicologia, em condições adequadas de privacidade. Durante cada encontro, foram fornecidos novos esclarecimentos sobre a pesquisa e realizada a leitura em voz alta e a assinatura do Termo de Consentimento Livre e Esclarecido, o que foi seguido pela aplicação do QUASHI. As respostas ao questionário foram gravadas em áudio e, posteriormente, transcritas na íntegra para proceder à análise dos relatos verbais obtidos.

Em um segundo momento, cada idoso foi novamente contatado para a aplicação dos questionários de avaliação de qualidade de vida da OMS -WHOQOL-BREF e WHOQOL-OLD, o que ocorreu de forma individual, em dia e horário de conveniência para os participantes. Cumprida essa etapa, foi dado início à intervenção grupal, que foi composta por nove sessões temáticas psicoeducativas, com periodicidade semanal e duração aproximada de 100 minutos cada uma. O objetivo da intervenção consistiu em favorecer a ampliação ou o fortalecimento do repertório de autocuidado e de busca dos serviços de saúde, bem como a modificação de comportamentos de risco para agravos à saúde.

Como estratégias de condução do grupo, buscou-se estimular (1) a troca de experiências e a expressão de percepções e sentimentos; (2) a busca conjunta de soluções diante de obstáculos relativos à emissão de comportamentos promotores de saúde; e (3) a percepção de autoeficácia acerca das potencialidades para cuidar da própria saúde.
Em linhas gerais, foram fornecidas e discutidas informações acerca de cuidados necessários para prevenir, diagnosticar precocemente ou controlar doenças e melhorar a saúde, assim como informações sobre os limites e as potencialidades do envelhecimento e os fatores determinantes da saúde dos homens. Conforme o planejamento de cada sessão, as seguintes estratégias foram utilizadas nos encontros: fornecimento de informações por meio de estratégias orais (exposição dialogada com recursos audiovisuais); discussões em grupo; uso de materiais educativos escritos ou em vídeo; dramatizações, vivências e técnicas de dinâmica de grupo.

Ao término da intervenção, dois novos encontros individuais foram conduzidos com cada um dos idosos, ocorridos uma semana (avaliação pós-intervenção) e quatro meses após as sessões psicoeducativas (avaliação de seguimento). Nessas ocasiões, foram reaplicados os questionários de qualidade de vida e utilizada uma versão simplificada do QUASHI.

\section{Análise de dados}

As avaliações dos participantes acerca do próprio estado de saúde e suas concepções de saúde e de envelhecimento saudável foram categorizadas, conforme o modelo de Bardin (2002), de modo a compreender como eles percebiam a saúde pessoal no momento atual do ciclo de vida e quais os significados que atribuíam ao ter saúde de um modo geral e, mais especificamente, na velhice. Inicialmente, foram realizadas leituras repetidas do material transcrito e, em seguida, os relatos dos participantes foram recortados e agrupados tendo por base as similaridades percebidas em cada um dos eixos de análise, possibilitando a elaboração de categorias temáticas. Falas ilustrativas, acompanhadas de nomes fictícios dos participantes, foram selecionadas para favorecer a compreensão das categorias identificadas.

Os resultados dos instrumentos que avaliam qualidade de vida foram analisados quantitativamente, com uso do software Statistical Package for Social Sciences (SPSS), versão 18.0. Partindo de pontuações entre 1 e 5 para as questões que compõem os diferentes domínios avaliados, os escores foram calculados em escalas de 4 a 20, que foram transformadas em escalas de 0 a 100, tal como proposto nas regras para composição dos escores desses instrumentos. A verificação da ocorrência de diferenças nos escores médios obtidos em cada domínio, antes e depois das intervenções, foi efetuada com emprego do Teste Não Paramétrico de Friedman, que 
permite comparação de médias com medidas repetidas, delineamento do presente estudo.

\section{Resultados}

\section{Saúde autoavaliada}

Como representação do estado pessoal de saúde, predominou entre os participantes uma apreciação positiva acerca das suas condições atuais. $\mathrm{Na}$ escala de cinco pontos utilizada, não houve menção à saúde pessoal no nível mais baixo (péssima) em nenhum dos três momentos de coleta de dados da pesquisa. Ao longo das entrevistas, verificou-se uma frequência maior de respostas indicativas de uma autoavaliação da saúde como "boa", correspondente ao nível quatro da escala. A boa saúde foi assim avaliada, tomando-se como parâmetro principal a própria idade, com ênfase na ausência ou controle de agravos à saúde usuais na velhice. Paralelamente, a não indicação da saúde como "ótima" por esses senhores foi justificada pela existência de doenças ou dores associadas ao avançar da idade, as quais, embora sob controle, provocavam limitações e, assim, não lhes permitiam considerar a saúde como pertencente ao nível mais elevado da escala.

As sinalizações da saúde pessoal como "ótima" tiveram como motivos predominantemente alegados a ausência de problemas físicos limitadores, bem como a própria vitalidade, a manutenção de uma vida saudável e o prazer sentido em viver. Por outro lado, as apreciações da saúde como "regular" foram atribuídas, em especial, à presença de doenças e à instabilidade no quadro clínico.

Comparando os três momentos de avaliação, observou-se a manutenção do nível de saúde autopercebido de cinco participantes, havendo alterações no relato dos demais quanto a tal resposta. A saúde pessoal foi mais bem avaliada, na segunda ou na terceira entrevista por quatro idosos, enquanto outros quatro apresentaram decréscimo nesta avaliação, o que esteve associado a estressores referentes a problemas financeiros ou a acentuação de conflitos familiares.

$\mathrm{Na}$ primeira entrevista, pouco mais da metade dos participantes $(53,8 \%)$ avaliou-se como tão sadios quanto outras pessoas com idades equivalentes. $\mathrm{O}$ restante deles $(46,2 \%)$ avaliou que as pessoas acima de sessenta anos são, em geral, menos sadias do que eles próprios, indicando acreditarem ter um nível de saúde acima da média para esta faixa de idade. Tal consideração positiva da própria saúde em comparação aos demais idosos foi atribuída, principalmente, a um maior estado de ânimo percebido em si mesmo. Nenhum participante, portanto, avaliou a saúde pessoal de modo desfavorável em relação ao grupo de pares.

Em comparação a quando mais jovens, oito participantes julgaram-se como menos sadios, justificando o decréscimo na avaliação da saúde pessoal ao declínio físico ou cognitivo e ao surgimento de doenças, com suas complicações e restrições características, como pode ser verificado nos relatos a seguir: "a gente vai ficando de idade, a gente não tem aquela mente mais pra resolver certas coisas" (Amadeu, 76 anos); "eu fui sentir doença depois dos 62 anos, antes eu não sentia doença nenhuma, é coisa de velho mesmo" (Jânio, 71 anos); "podia comer tudo, podia beber tudo, podia correr bastante, podia passar a noite sem dormir, hoje eu não posso" (Eusébio, 63 anos). Em geral, esses idosos ressaltaram as perdas físicas, sendo que cognitiva e emocionalmente relataram sentir-se ainda semelhantes a épocas passadas do ciclo de vida, como atesta o depoimento de Oscar (70 anos): "Assim, de espírito, eu me sinto como quando eu tinha 30 anos. Só que fisicamente eu não tenho mais condições. [...] aí, às vezes, eu quero avançar demais e... opa, tem que parar aí velho".

Por outro lado, três idosos julgaram suas condições de saúde presentes como melhores do que em fases anteriores do desenvolvimento, atribuindo esse fato, sobretudo, a um estilo de vida atual mais sadio, com abandono de hábitos nocivos à saúde e maior assertividade. Dois idosos não fizeram distinção entre a saúde na velhice e quando mais jovens, considerando seus status de saúde como equivalentes nos dois períodos da vida.

Significados atribuidos ao "ter saúde" e ao "envelhecimento saudável"

A saúde foi apontada pelos participantes como um valor primordial da vida. A categorização de suas respostas acerca dos sentidos conferidos ao "ter saúde" revelou que a mesma foi tomada por eles em várias perspectivas. Verificou-se referências tanto a aspectos biológicos como a aspectos psicossociais do conceito. Nas respostas com ênfase na dimensão física, a saúde foi destacada como ausência de sinais e sintomas (p. ex.: "não sentir dores") e como a existência de condições físicas para manter atividades, dentre elas o trabalho e a possibilidade de andar, nadar e correr. $\mathrm{Na}$ dimensão psicossocial, predominante antes e após a intervenção, a saúde foi compreendida como autorregulação afetiva e cognitiva, incluindo disposição e ânimo para trabalhar e divertir-se, controle emocional, bom convívio social e 
capacidade para resolver ou enfrentar problemas, conforme ilustram os seguintes relatos: "viver bem com a gente mesmo"; "ter alegria pra levantar, brincar, ter satisfação em participar das coisas da vida"; "não se estressar com qualquer coisa"; "ter prazer em viver em comunidade". Foi descrita ainda como manutenção de hábitos saudáveis (p. ex., "boa alimentação, atividade física e mental") e ausência de hábitos nocivos (p. ex., "não fumar").

Em relação aos determinantes do envelhecimento saudável ou bem-sucedido, os conteúdos relatados pelos entrevistados foram também agrupados em fatores físicos e fatores psicossociais. Dentre os fatores físicos mencionados, foram identificadas duas subcategorias, que apresentavam em comum o fato de enfatizarem as necessidades ou limitações orgânicas da pessoa idosa, a saber: a busca de serviços médicos e uso de medicação (p. ex., "tem que procurar o médico, fazer os exames") e o reconhecimento e respeito às próprias limitações físicas (p. ex., "viver a vida, mas sem arriscar, sem passar dos limites").

Os fatores psicossociais, por sua vez, foram organizados em três subcategorias: 1. manutenção de hábitos saudáveis (p. ex., "boa alimentação, muito exercício físico, porque depois dos 50 tem que puxar mais") e ausência de hábitos nocivos à saúde (p. ex., "não beber"); 2. autorregulação afetiva e cognitiva, incluindo manutenção de atividades de trabalho ou recreativas (p. ex., "trabalhar é o que mais segura a pessoa quando envelhece", "se divertir, se ocupar em um lazer qualquer que goste", "uma viagem faz bem, uma boa leitura"), manejo de situações estressantes (p. ex., "procurar não ter muita preocupação"), satisfação em viver (p. ex., "ter amor pela vida"), bom relacionamento familiar (p. ex., "o mais importante é a família, a gente viver bem”); prática de ações solidárias (p. ex., uma palavra amiga, que dá força, uma visita a um hospital, a um asilo, que faça alguém se sentir bem"); e 3. o controle das finanças (p. ex., "controlar a vida financeira pra não ter problema de ultrapassar o limite do banco").

De um modo geral, os idosos ressaltaram ser necessário um conjunto de fatores para assegurar um bom envelhecer, com predomínio daqueles de natureza psicossocial. Após a intervenção, todos eles destacaram, em seus depoimentos, a necessidade de bons hábitos para o alcance de um envelhecimento saudável.

\section{Avaliação da qualidade de vida}

Os participantes, de acordo com os resultados do WHOQOL-OLD, mostraram uma avaliação predominantemente positiva de sua qualidade de vida. A maior parte deles (dez casos) alcançaram escores globais superiores a 70,0 desde a primeira avaliação. Após as intervenções, houve estabilidade nos escores obtidos, com uma tendência discreta para aumento. Onze participantes apresentaram escores mais elevados na segunda e/ou na terceira aplicação do instrumento, realizadas após a intervenção (Tabela 1).

Analisando o grupo, em relação às médias dos escores das seis facetas do WHOQOL-OLD, a Tabela 2 revela que, nos momentos pós-intervenção, houve diferenças significativas, de acordo com o Teste Friedman, apenas para Participação Social $(p=0,014)$, que inclui satisfação com o modo de usar o tempo, com o nível de atividade e com oportunidades para participação na comunidade. As médias das demais facetas e do escore global não apresentaram diferenças estatisticamente significativas, embora tenham ocorrido

Tabela 1. Médias, desvios padrão e valores do Teste de Friedman das Facetas e Escores Globais do WHOQOL-OLD

\begin{tabular}{lccccc}
\hline Facetas e escore global & $\begin{array}{c}\text { Linha } \\
\text { de base } \\
\text { M/DP }\end{array}$ & $\begin{array}{c}\text { Pós-intervenção } \\
\text { M/DP }\end{array}$ & $\begin{array}{c}\text { Seguimento } \\
\text { M/DP1 }\end{array}$ & $\begin{array}{c}\text { Teste } \\
\text { Friedman }^{1}\end{array}$ & $p$ \\
\hline Funcionamento do sensório & $80,7 / 18,6$ & $83,6 / 15,2$ & $74,4 / 28,1$ & 3,063 & 0,216 \\
Autonomia & $54,3 / 14,1$ & $61,5 / 15,9$ & $59,1 / 17,7$ & 5,692 & 0,058 \\
Ativ. passadas, Presentes, futuras & $70,2 / 17,7$ & $73,1 / 17,7$ & $70,4 / 16,1$ & 2,167 & 0,338 \\
Participação social & $67,3 / 14,7$ & $69,2 / 20,8$ & $78,4 / 8,1$ & 8,600 & $0,014^{*}$ \\
Morte e morrer & $68,7 / 20,2$ & $68,3 / 22,0$ & $69,3 / 27,3$ & 0,813 & 0,666 \\
Intimidade & $74,0 / 15,3$ & $73,1 / 18,5$ & $72,7 / 16,1$ & 2,294 & 0,818 \\
Escore global & $75,4 / 9,0$ & $77,2 / 11,2$ & $76,6 / 9,1$ & 1,756 & 0,416 \\
\hline
\end{tabular}

${ }^{1} N=11$, dois participantes não fizeram avaliação no seguimento; ${ }^{*} p<0,05$. 
aumentos nos escores relativos a Habilidades Sensoriais, Passado, Presente e Futuro, Morte e Morrer e, sobretudo, Autonomia.

No WHOQOL-BREF, os idosos indicaram percepções satisfatórias de qualidade de vida no panorama geral. Na Tabela 2, estão apresentados os níveis de satisfação com a qualidade de vida nos quatro domínios do WHOQOL-BREF e no escore global, considerando as médias do grupo. Os níveis de satisfação mais elevados foram relativos aos domínios Relações Sociais e Físico. Por outro lado, os escores mais baixos foram referentes ao domínio Psicológico, que variaram de 51,9 a 53,8. Diferenças estatisticamente significativas não foram observadas entre os idosos em nenhum desses domínios por ocasião da comparação das médias obtidas na aplicação do questionário antes e após as intervenções.

\section{Discussão}

Apesar da grande incidência de doenças crônicas entre os participantes, seus relatos tornaram nítida a predominância da autopercepção positiva do estado pessoal de saúde, com um maior número deles avaliando sua saúde como "boa". Eles reconheceram limitações na saúde pessoal, associando-as a declínios físicos e/ ou cognitivos e ao surgimento de enfermidades, mas também destacaram capacidades e habilidades surgidas ou aperfeiçoadas no momento de vida atual. Todos eles se avaliaram como tão ou mais sadios do que seus pares e alguns se perceberam inclusive como detentores de saúde igual ou melhor do que quando mais jovens.

De modo similiar, outros estudos também têm evidenciado a predominância de autopercepção da saúde positiva e de satisfação com a própria vida entre idosos (por ex.: Assis \& et al., 2009; Ribeiro, Neri, Cupertino, \& Yassuda, 2009; Vitorino, 2012), sendo que um deles, realizado exclusivamente com homens, revelou que a maioria dos entrevistados avaliou ter uma boa saúde e $40,7 \%$ consideraram seu estilo de vida como frequentemente saudável (Loeb, 2003). Influências de gênero e idade têm sido constatadas na autoavaliação da saúde. As mulheres tendem a avaliar o estado de saúde pessoal de modo mais negativo em comparação aos homens e a pessoas mais jovens (Alves \& Rodrigues, 2005; De Vitta, Neri, \& Padovani, 2006; Lee, 2000; Pinheiro, Viacava, Travassos, \& Brito, 2002).

Vários fatores influenciam a autopercepção do estado de saúde na velhice. A partir de um estudo conduzido com 2.135 idosos na cidade de São Paulo, Alves e Rodrigues (2005) identificaram quatro grupos de determinantes da autopercepção: o demográfico, o socioeconômico, a presença de doenças crônicas e a capacidade funcional. Supõe-se, na presente pesquisa, que a autoavaliação da saúde pessoal esteve relacionada a uma gama de fatores clínicos e sociais positivos, como o controle da sintomatologia de doenças, a preservação da capacidade funcional, a manutenção de hábitos de vida saudáveis, o suporte familiar e as oportunidades de participação social e de novas aprendizagens acadêmicas.

A avaliação subjetiva de saúde tem sido frequentemente apontada como uma medida válida para predizer resultados futuros de saúde entre pessoas idosas, sendo reconhecida como um preditor importante de mortalidade e de declínio funcional (Lee, 2000). Além disso, a avaliação feita pela pessoa acerca das suas condições de saúde pode refletir seu autoconhecimento de problemas físicos e psicológicos e evidenciar sua satisfação com a própria saúde, tornando-a apta a comunicar ao profissional da saúde seus sintomas e dificuldades.

Tanto nas entrevistas para levantamento da linha de base como nas entrevistas pós-intervenção, os idosos

Tabela 2. Médias, desvios padrão e Valores do Teste de Friedman dos Domínios e Escores Globais do WHOQOL-BREF

\begin{tabular}{lccccc}
\hline Domínios e escore global & $\begin{array}{c}\text { Linha } \\
\text { de base } \\
\text { M/DP }\end{array}$ & $\begin{array}{c}\text { Pós } \\
\text { Intervenção } \\
\text { M/DP }\end{array}$ & $\begin{array}{c}\text { Seguimento } \\
\text { M/DP1 }\end{array}$ & $\begin{array}{c}\text { Teste } \\
\text { Friedman }^{1}\end{array}$ & $p$ \\
\hline Físico & $71,4 / 14,7$ & $76,9 / 10,6$ & $75,9 / 11,3$ & 0,50 & 0,97 \\
Psicológico & $52,6 / 6,9$ & $51,9 / 7,9$ & $53,8 / 6,0$ & 1,135 & 0,57 \\
Relações Sociais & $66,7 / 14,8$ & $73,1 / 13,7$ & $73,5 / 15,3$ & 2,389 & 0,30 \\
Meio Ambiente & $63,2 / 16,8$ & $66,3 / 9,3$ & $65,9 / 11,9$ & 1,366 & 0,50 \\
Escore Global & $75,0 / 19,7$ & $79,8 / 10,9$ & $77,3 / 20,8$ & 2,515 & 0,284 \\
\hline
\end{tabular}

${ }^{1} N=11$, dois participantes não fizeram avaliação no seguimento.

Psico-USF, Bragança Paulista, v. 19, n. 3, p. 421-431, set./ deæ. 2014 
demonstraram que, para eles, o ter saúde estava vinculado não somente à ausência de sintomas de doenças ou à manutenção de boas condições físicas, mas também à maneira de viver e de vislumbrar a vida. Destaca-se, neste último aspecto, a capacidade de implementar ações em prol do próprio bem-estar, abarcando a emissão de comportamentos preventivos, a manutenção de atitudes positivas diante da vida, a busca do controle emocional e o convívio social. Essas constatações sugerem que, atualmente, a saúde vem cada vez mais sendo tomada em várias perspectivas e concebida como uma integração de múltiplos fatores. Muitos idosos têm revelado uma visão ampla e apropriada dos cuidados necessários para a preservação da saúde e têm adotado uma postura ativa quanto à possibilidade de atuarem na manutenção ou melhoria da sua condição pessoal. As intervenções psicoeducativas contribuem para fortalecer esta compreensão da saúde como uma realidade ampla e integradora, o que se mostra de grande relevância, dada a possibilidade de se contribuir para a desconstrução de uma imagem passiva da velhice, como centrada em perdas inevitáveis.

A compreensão dos componentes do envelhecimento saudável na perspectiva dos próprios sujeitos mostra-se necessária para a definição de metas pessoais, considerando o que eles próprios julgam ainda ser necessário praticar para o alcance de uma velhice saudável. Conforme advogam Miller e Iris (2002), o desenvolvimento de programas de promoção de saúde para idosos deve levar em consideração os significados, valores e metas da perspectiva deles próprios.

Em relação às medidas de qualidade de vida, a maioria dos participantes alcançou níveis elevados nas facetas do WHOQOL-OLD e nos domínios do WHOQOL-BREF. A literatura mostra benefícios de atividades educativas em vários domínios da qualidade de vida mediante a análise de dados obtidos antes e após intervenções com idosos. Castro et al. (2007), por exemplo, encontraram diferenças estatisticamente significativas nos domínios psicológico e meio ambiente do WHOQOL-BREF, ao passo que Ascensão (2011) verificou alterações significativas em todos os domínios do instrumento. No estudo de Vitorino (2012), os indicadores de qualidade de vida medidos pelo WHOQOL-OLD evidenciaram diferenças significativas após a intervenção nas facetas Presente, Passado e Futuro, Participação Social e Intimidade.

No presente estudo, as médias do WHOQOL-OLD referentes à participação social mostraram que os escores pós-intervenção atingiram patamares acima daqueles da avaliação pré-intervenção, indicando uma melhora no nível de satisfação nesta faceta. Este dado, em conjunto com os relatos acerca dos ganhos sociais possibilitados pela participação nas sessões psicoeducativas, reforça o valor da manutenção ou ampliação das relações sociais para pessoas idosas, bem como confirma o quanto os trabalhos grupais tendem a lhes fornecer um ambiente rico de oportunidades para novas interações e formas de engajamento social (Ascensão, 2011; Garcia, Yagi, Souza, Odoni, Frigério, \& Merlin, 2006; Lahm-Vieira \& Boeckel, 2012; Magesky, Modesto, \& Torres, 2009; Morais, 2009).

Por outro lado, o domínio psicológico no WHOQOL-BREF foi responsável pelos escores mais baixos entre os idosos, o que aponta a necessidade de se atentar para o estado psicológico de pessoas nesta faixa de idade. Pressupõe-se que resultados mais significativos neste domínio requeiram intervenções mais específicas e por maior período de tempo do que foi alvo de investimento nesta pesquisa. Diante disso, sugere-se que estudos posteriores possam avaliar intervenções psicológicas com homens idosos direcionadas ao melhoramento da autoestima e da regulação emocional.

Investigações sobre qualidade de vida que se baseiam na percepção dos próprios idosos são necessárias para subsidiar programas e fomentar políticas voltadas para a promoção da saúde nessa população. Nesse propósito, o WHOQOL-OLD e o WHOQOL-BREF revelaram-se instrumentos abrangentes e de aplicação simples para aferir a qualidade de vida em idosos.

O presente estudo contribui para ressaltar efeitos positivos de intervenções grupais com idosos, em especial a reflexão sobre temas relacionados ao envelhecimento saudável e o aumento em indicadores de autocuidado, o que condiz com resultados alcançados por outras intervenções destinadas a esta população (Assis et al., 2009; Cervato et al., 2005; Dapp et al., 2005; Morais, 2009), mas com o diferencial de ter contado exclusivamente com homens.

\section{Considerações finais}

Ao focalizar homens, o presente trabalho permitiu verificar como eles estão se engajando em espaços de promoção de saúde e forneceu uma compreensão de experiências de envelhecimento ativo entre idosos do sexo masculino. $\mathrm{O}$ autorrelato dos participantes sobre temas variados relacionados à saúde permitiu um mapeamento de temáticas que se centram na descrição 
de necessidades de saúde e interesses do ponto de referência de homens idosos. Desse modo, os resultados obtidos podem servir como dados exploratórios para o planejamento de pesquisas e intervenções futuras.

No entanto, além de buscar diferenças entre os gêneros, é necessário reconhecer diferentes formas e estilos de ser homem. O trabalho de promoção da saúde não pode se basear na suposição de um conjunto de características iguais que leva a interpretar os homens como um grupo composto por valores e comportamentos comuns e generalizáveis. Suas práticas de saúde dependem de vários fatores demográficos, pessoais e culturais e, como tal, são diversas.

Algumas limitações desta pesquisa necessitam ser apontadas, a começar pela quantidade pequena de participantes da intervenção $(n=13)$, os quais apresentavam um alto nível de motivação para o tipo de atividade realizada, além de já constituírem, em sua maioria, bons cuidadores da própria saúde. Esses aspectos impedem a generalização dos resultados a outros idosos. Ademais, a avaliação da intervenção ocorreu sem a utilização de um grupo controle e os instrumentos utilizados não permitiram realizar uma quantificação mais precisa dos seus efeitos.

Em relação a pesquisas futuras, sugere-se a realização de estudos que tenham por objetivo comparar os efeitos de intervenções psicoeducativas sobre comportamentos e autopercepções de saúde e qualidade de vida de homens e mulheres idosos, assim como de homens de diferentes faixas de idade.

\section{Referências}

Alves, L. S., \& Rodrigues, R. N. (2005). Determinantes da autopercepção de saúde entre idosos do Município de São Paulo, Brasil. Revista Panamericana de Salud Publica, 17(5/6), 333-341.

Ascensão, M. C. de A. (2011). Solidão, depressão e qualidade de vida no idoso: implementação de um programa de intervenção (Dissertação de Mestrado não publicada), Faculdade de Psicologia da Universidade de Lisboa, Portugal.

Assis, M. de, Hartz, Z. M. A., \& Valla, V. V. (2004). Programas de promoção da saúde do idoso: uma revisão da literatura científica no período de 1990 a 2002. Ciência \& Saúde Coletiva, 9(3), 557-581.

Assis, M. de, Hartz, Z. M. de A., Pacheco, L. C., \& Valla, V. V. (2009). Avaliação do projeto de promoção da saúde do Núcleo de Atenção ao Idoso: um estudo exploratório. Interface - Comunicação, Saúde, Educação, 13(29), 367-382.

Bardin, L. (2002). Análise de conteúdo (L. A. Reto \& A. Pinheiro, trads.). Lisboa: Edições 70. (Original publicado em 1977).

Bode, C., \& Ridder, D. T. D. (2007). Investing in the future - identifying participants in an educational program for middle-aged and older adults. Health Education Research, 22(4), 473-482.

Camarano, A. M. (2002). Envelhecimento da população brasileira: Uma contribuição demográfica. Em E. V. Freitas, L. Py, A. L. Neri, F. A. X. Cançado, M. L. Gorzoni \& S. M. Rocha (Eds.), Tratado de Geriatria e Gerontologia (pp. 58-71). Rio de Janeiro: Guanabara Koogan.

Carvalho, C. M. R. G. de, Fonseca, C. C. C., \& Pedrosa, J. I. (2004). Educação para a saúde em osteoporose com idosos de um programa universitário: repercussões. Cadernos de Saúde Pública, 20(3), 719-726.

Castro, P. C., Tahara, N., Rebelatto, J. R., Driusso, P., Aveiro, M. C., \& Oishi, J. (2007). Influência da Universidade Aberta da Terceira Idade (UATI) e do Programa de Revitalização (REVT) sobre a qualidade de vida de adultos de meia-idade e idosos. Revista Brasileira de Fisioterapia, 11(6), 461-467.

Cervato, A. M., Derntl, A. M., Latorre, M. do R. D. de O., \& Marucci, M. de F. N. (2005). Educação nutricional para adultos e idosos: uma experiência positiva em Universidade Aberta para a Terceira Idade. Revista de Nutrição, 18(1), 41-52.

Dalla Vecchia, R., Ruiz, T., Bocchi, S. C. M., \& Corrente, J. E. (2005). Qualidade de vida na terceira idade: um conceito subjetivo. Revista Brasileira de Epidemiologia, 8(3), 246-252.

Dapp, U., Anders, J., von Renteln-Kruse, W., \& MeierBaumgartner, H. P. (2005). Active health promotion in old age: methodology of a preventive intervention programme provided by an interdisciplinary health advisory team for independent older people. Journal of Public Health, 13(3), 122-127.

De Vitta, A., Neri, A. L., \& Padovani, C. R. (2006). Saúde percebida em homens e mulheres sedentários e ativos, adultos jovens e idosos. Salusvita, 25(1), 23-34.

Diogo, M. J. D., Ceolim, M. F., \& Cintra, F. A. (2000). Implantação do Grupo de Atenção à Saúde do 
Idoso (GRASI) no Hospital de Clínicas da Universidade Estadual de Campinas (SP): Relato de experiência. Revista Latino-Americana de Enfermagem, 8(5), 85-90.

Domínguez, M. C. P., \& Juste, M. R. J. (2003). Intervención educativa para la mejora de la calidad de vida del paciente geriátrico. Un programa interdisciplinar en educación social. Revista Portuguesa de Pedagogía, 37(3), 231-251.

Fleck, M. P., Chachamovich, E., \& Trentini, C. (2006). Desenvolvimento e validação da versão em português do módulo WHOQOL-OLD. Revista de Saúde Pública, 40(5), 785-791.

Fleck, M. P. A., Louzada, S., Xavier, M., Chachamovich, E., Vieira, G., Santos, L., \& Pinzon, V. (2000). Aplicação da versão em português do instrumento WHOQOL-bref. Revista de Saúde Pública, 34(2), 178-183.

Garcia, M. A. A., Yagi, G. H., Souza, C. S., Odoni, A. P. C., Frigério, R. M., \& Merlin, S. S. (2006). Atenção à saúde em grupos sob a perspectiva dos idosos. Revista Latino-Americana de Enfermagem, 14(2), 175-182.

Gonçalves, L. H. T., \& Schier, J. (2005). Grupo Aqui e Agora: uma tecnologia leve de ação sócio-educativa de enfermagem. Texto \& Contexto Enfermagem, 14(2), 271-279.

Lahm-Vieira, C. R., \& Boeckel, M. G. (2012). (Qual) idade de vida: intervenção psicológica junto a grupo da terceira idade. Estudos Interdisciplinares sobre o Envelhecimento, 17(1), 183-199.

Lee, Y. (2000). The predictive value of self assessed general, physical, and mental health on functional decline and mortality in older adults. Journal of Epidemiology and Community Health, 54(2), 123-129.

Loeb, S. J. (2003). The Older Men's Health Program and Screening Inventory: a tool for assessing health practices and beliefs. Geriatric Nursing, 24(5), 278-285.

Magesky. A. M, Modesto, J. L., \& Torres, L. C. (2009). Intervenção psicossocial com um grupo de idosos institucionalizados. Revista Saúde e Pesquisa, 2(2), 217-224.

Miller, A. M., \& Iris, M. (2002). Health promotion attitudes and strategies in older adults. Health Education \& Behavior, 29(2), 249-267.
Morais, O. N. P. de (2009). Grupos de idosos: atuação da psicogerontologia no enfoque preventivo. Psicologia: Ciência e Profissão, 29(4), 846-855.

Neri, A. L, Yassuda, M. S., \& Cachioni, M. (2004). Velhice bem sucedida: aspectos afetivos e cognitivos. Campinas: Papirus.

Netto, M. P. (2002). O Estudo da velhice no século XX: histórico, definição do campo e termos básicos. Em E. V. Freitas, L. Py, A. L. Neri, F. A. X. Cançado, M. L. Gorzoni \& S. M. Rocha (Eds.), Tratado de geriatria e gerontologia (pp. 2-12). Rio de Janeiro: Guanabara Koogan.

Oliffe, J. L., Gerbrandt, J., Bottorff, J. L., \& Hislop, T. G. (2010). Health promotion and illness demotion at prostate cancer support groups. Health Promotion Practice, 11(4), 562-571.

Pinheiro, R. S., Viacava, F., Travassos, C., \& Brito, A. S. (2002). Gênero, morbidade, acesso e utilização de serviços de saúde no Brasil. Ciência \& Saúde Coletiva, 7(4), 687-707.

Ribeiro, P. C. C., Neri, A. L., Cupertino, A. P. F. B., \& Yassuda, M. S. (2009). Variabilidade no envelhecimento ativo segundo gênero, idade e saúde. Psicologia em Estudo, 14(3), 501-509.

Seidl, E. M. F., \& Zannon, C. M. L. da C. (2004). Qualidade de vida e saúde: aspectos conceituais e metodológicos. Caderno de Saúde Pública, 20(2), 580-588.

Silva, H. S., Lima, A. M. M., \& Galhardoni, R. (2010). Successful aging and health vulnerability: approaches and perspectives. Interface - Comunicação, Saúde, Educação, 14(35), 867-877.

Vitorino, S. dos S. (2012). Qualidade de vida percebida por idosos de um programa educativo: avaliação de uma intervenção psicológica (Dissertação de Mestrado não publicada), Programa de Mestrado em Ciências do Envelhecimento da Universidade São Judas Tadeu, São Paulo, Brasil.

World Health Organization - WHO (2005). Envelhecimento ativo: uma política de saúde. Brasília: Organização Pan-Americana da Saúde. Disponível em: http:// bvsms.saude.gov.br/bvs/publicacoes/envelhecimento_ativo.pdf

Recebido em: 11/04/2013

Reformulado em: 17/01/2014

Segunda reformulação em: 20/02/2014

Aprovado em: 23/04/2014 
Sobre as autoras:

Lilian Maria Borges graduou-se em Psicologia pela Universidade Federal do Pará e realizou o mestrado e o doutorado na área pelo Programa de Pós-Graduação em Processos de Desenvolvimento Humano e Saúde da Universidade de Brasília. Atualmente, exerce o cargo de professor adjunto do Instituto de Psicologia da Universidade Federal Rural do Rio de Janeiro, tendo integrado anteriormente o corpo docente do curso de Psicologia da Universidade Católica de Brasília.

Eliane Maria Fleury Seidl possui graduação, mestrado e doutorado em Psicologia pela Universidade de Brasilia e pós-doutorado pela Universidade Federal de Santa Catarina. É professora associada da Universidade de Brasilia, onde integra o corpo docente dos Programas de Pós-Graduação em Processos de Desenvolvimento Humano e Saúde (Instituto de Psicologia) e em Bioética (Faculdade de Ciências da Saúde). Possui bolsa de produtividade em pesquisa do CNPq e coordena, atualmente, o GT Psicologia da Saúde nas Instituições e na Comunidade junto à ANPEPP. E-mail: seidl@unb.br.

Contato com as autoras:

UFRRJ, Instituto de Psicologia. Km 7, BR 465

CEP 23890-000. Seropédica, RJ.

E-mail: limaborgesg@gmail.com. 
